\title{
2020 emerging investigator issue of Science China Chemistry
}

\author{
Li-Jun $\operatorname{Wan}^{1,2^{*}}$ \\ ${ }^{1}$ Institute of Chemistry, Chinese Academy of Sciences, Beijing 100190, China; \\ ${ }^{2}$ University of Chinese Academy of Sciences, Beijing 100049, China
}

Received September 1, 2020; accepted September 1, 2020; published online September 4, 2020

Citation: Wan LJ. 2020 emerging investigator issue of Science China Chemistry. Sci China Chem, 2020, 63: 1331-1335, https://doi.org/10.1007/s11426-0209861-7

Emerging Investigator issue features the works conducted by young scientists in the earlier stage of their independent research career, with the potentials to influence the direction in chemistry and related fields. The contributors are selected from the recommendation of senior scientists as well as the editorial board members of Science China Chemistry. 17 papers in the issue include 6 Reviews, 1 Communication and 10 Articles, mainly covering the topics on asymmetric synthesis with $\mathrm{CO}_{2}$, organic solar cells, covalent/metal organic frameworks, rechargeable $\mathrm{Li} / \mathrm{Na}$ batteries, photocatalysis, electrocatalysts, bioimaging and photodynamic therapy, 2D conjugated porous polymers, ultralong organic phosphorescence materials, aggregation-induced emission materials, and single-molecule protein analysis.

The profiles of each invited author are briefly shown here. Sincerely wishes to their success in future careers.

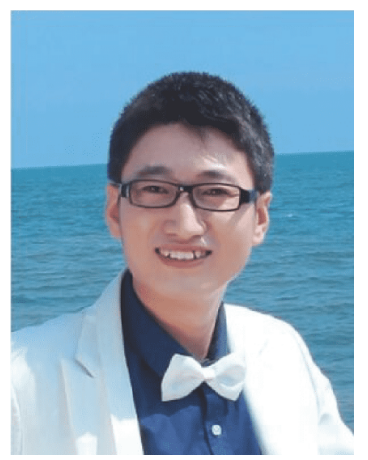

Dr. Zhongfu An graduated from Nanjing University of Post and Telecommunication and received his $\mathrm{PhD}$ degree in 2014. After graduation, he went to Department of Chemistry, National University of Singapore for his post-doctoral research. He joined Institute of Advanced Materials, Nanjing Tech University and started his independent research at the end of 2015. He was awarded excellent dissertation for doctor degree in Jiangsu Province (2015), the First prize of Natural Science Award of excellent achievements in scientific research of colleges and universities of the Ministry of Education (2018), etc. He focused on ultralong organic phosphorescence materials and their applications in organic optoelectronics and flexible electronics. He initially designed and synthesized multiple series of one-component, metal-free organic phosphorescent materials with ultralong emission lifetime ( $\sim$ second scale) under ambient condition.

*Corresponding author (email: wanlijun@iccas.ac.cn) 


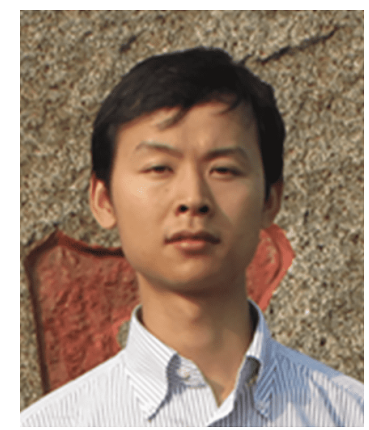

Dr. Long Chen received his $\mathrm{PhD}$ degree in 2009 at the Institute for Molecular Science (IMS, Japan). He then joined Max Planck Institute for Polymer Research (MPIP, Germany) as an Alexander von Humboldt research fellow. In March 2012, he was appointed as a project leader of the department of synthetic chemistry at MPIP. He officially joined Tianjin University as a full professor in September 2014. His current research focuses on the design and synthesis of $2 \mathrm{D}$ conjugated porous polymers for catalysis and energy-related applications.

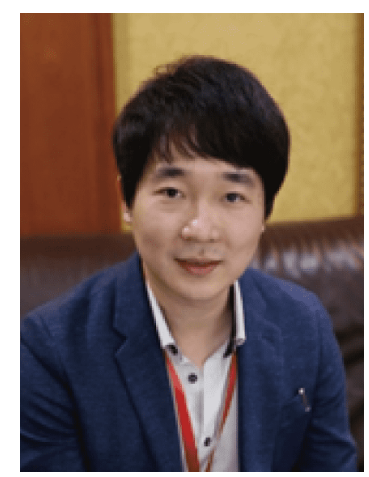

Dr. Dan Ding received his PhD degree from the Department of Polymer Science and Engineering in Nanjing University in 2010. After a postdoctoral training in the National University of Singapore, he joined Nankai University in March 2013, where he is currently a Professor in State Key Laboratory of Medicinal Chemical Biology, Key Laboratory of Bioactive Materials, Ministry of Education, and College of Life Science. He also conducted his work in The Hong Kong University of Science and Technology as a visiting scholar. His current research focuses on the design and synthesis of smart/functional molecular imaging probes and exploration of their biomedical applications.

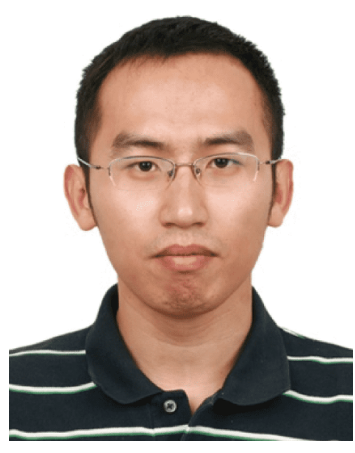

Dr. Xiao Feng received his BS degree in material chemistry in 2008 and $\mathrm{PhD}$ degree in material science in 2013 from Beijing Institute of Technology (BIT). He pursued his study in Japan as a joint $\mathrm{PhD}$ student at the Institute for Molecular Science (2009-2012). He started his academic career as an assistant professor at BIT (2013), and now he is a professor in School of Chemistry and Chemical Engineering at BIT. His current research interest mainly focuses on crystalline porous materials (i.e., covalent organic frameworks and metal-organic frameworks) and their applications in membrane separation (i.e., gas separation and desalination).

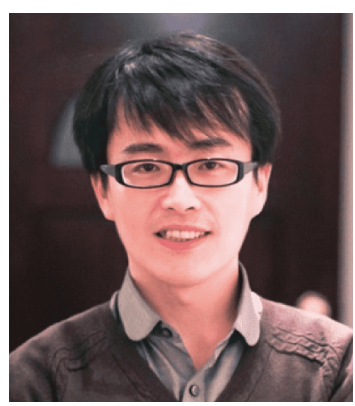

Dr. Lele Li is a professor in National Center for Nanoscience and Technology of China (NCNST). He received his $\mathrm{PhD}$ degree from Peking University in 2010 under the supervision of Prof. Chun-Hua Yan. After two years of postdoctoral research in Prof. Yi Lu's group at University of Illinois at Urbana-Champaign, he worked with Prof. Robert Langer and Prof. Daniel Kohane as a postdoctoral associate at MIT and Harvard Medical School from 2012-2016. Then, he started his own independent career at NCNST in 2016. His current research interest focuses on the development of novel technologies and tools for biosensing, bioimaging and disease treatment with high temporal and spatial precision. He is the recipient of the Thousand Talents Program Award for Young Researchers (2015) and National Science Fund for Excellent Young Scholars (2018). 


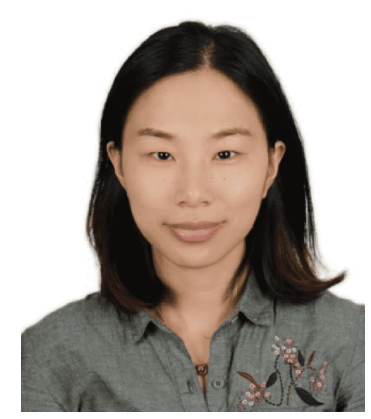

Dr. Qianqian Li received her BSc degree from Hubei University, China, in 2004 and then obtained her $\mathrm{PhD}$ degree under the supervision of Prof. Zhen Li at Wuhan University in 2009. After two years postdoctoral research at Department of Physics, she became a faculty at College of Chemistry and Molecular Sciences in Wuhan University, and was promoted to a full professor in 2018. In 2016-2017, she worked as a visiting scholar in the University of California, Los Angeles. Her research interest focuses on the design and synthesis of new opto-electronic functional materials.

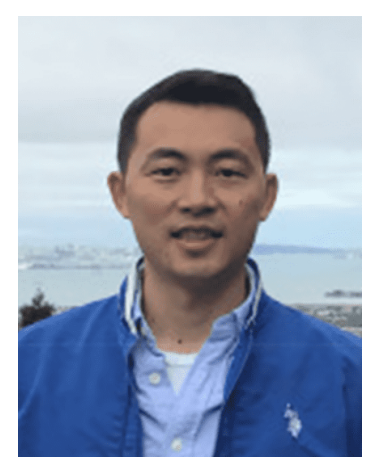

Dr. Wei Luo is a Professor in the School of Materials Science and Engineering at Tongji University. He received $\mathrm{PhD}$ degree in Materials Science from Huazhong University of Science and Technology in 2012. Prior to his current position, he worked as a postdoctoral researcher at Oregon State University (2012-2014), and University of Maryland (UMD, 2014-2016) until being promoted to Assistant Research Professor at UMD (2016-2017). His research interest includes energy storage and conversion devices, biomass materials, and low-dimensional nanomaterials.

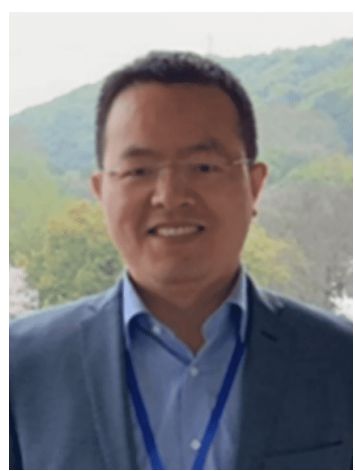

Dr. Jie Min is a full professor at the Institute for Advanced Studies of Wuhan University. He focused on the photovoltaic materials in the group of Prof. Yongfang Li as a Joint Master (2008-2011). In 2015 he completed his PhD study in the Institute of Materials for Electronics and Energy Technology (i-MEET) at the Friedrich Alexander University ErlangenNuernberg under the supervision of Prof. Christoph J. Brabec. From October 2015, he was a postdoctoral fellow in the group of Prof. Brabec in i-MEET. He joined the Wuhan University in 2017. His major research interest is in the physics and the chemistry of organic photovoltaic materials, and photovoltaic device physics and engineering.

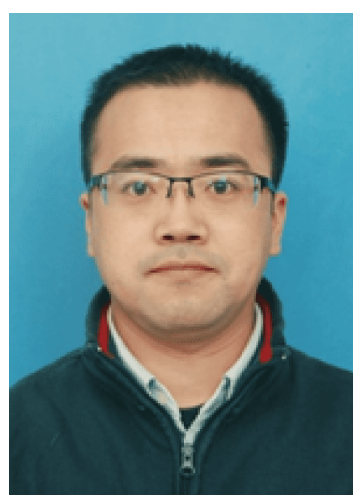

Dr. Yun-Xiang Pan received his BS degree in chemical engineering in 2004 from Hebei University of Technology, and $\mathrm{PhD}$ degree in environmental chemical engineering in 2010 from Tianjin University. From 2008 to 2009, he studied as a joint-training $\mathrm{PhD}$ student at the Southern Illinois University, Carbondale, USA. After that, as a post-doctor, he conducted his researches in Aarhus University, Denmark, Tianjin University and Nanyang Technological University, Singapore. In 2014, he joined Hefei University of Technology as a Huangshan Young Scholar. In 2018, he took the position in Shanghai Jiao Tong University. He is now a full professor in Department of Chemical Engineering, Shanghai Jiao Tong University. His research focuses on fabricating nanomaterials through novel room-temperature strategy for catalysis, electronics and biomedicine. 


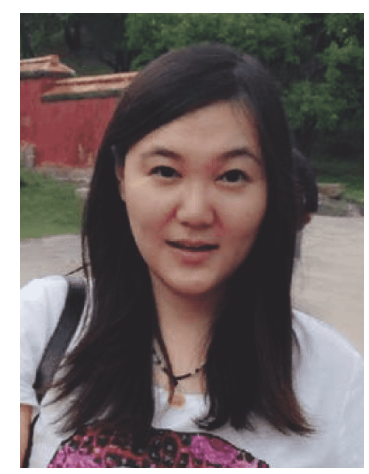

Dr. Jiashu Sun obtained her BS degree at Beijing Institute of Technology (2006), followed by a PhD degree (2010) from Vanderbilt University, working on microfluidics and single cell analysis. She joined the National Center for Nanoscience and Technology (NCNST) as an Assistant Professor in 2011 and was promoted to a Full Professor/Principal Investigator in 2016. Her research interest includes microfluidics for liquid biopsy, point-of-care testing, and nanomedicine. She was selected into the Excellent Young Scholars Program by NSFC (2016), Beijing Nova Program (2016), and Beijing Youth Talent Support Program (2018).

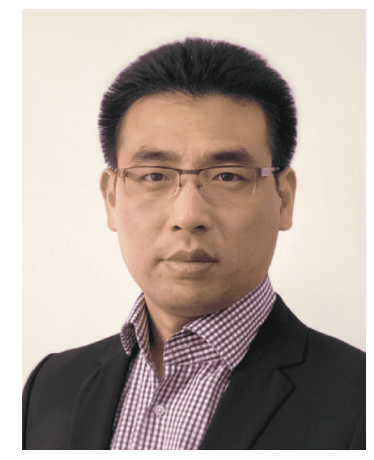

Dr. Tao Wang received his BSc degree in Polymer Materials (2002) and MSc degree in materials science (2005). He obtained his PhD degree in Soft Condensed Matter Physics from the University of Surrey (UK) in 2009, and then moved to the Department of Physics and Astronomy at the University of Sheffield (UK) where he worked as a post-doctor on organic solar cells. He was appointed a Professor in the School of Materials Science \& Engineering, Wuhan University of Technology (China) in 2013. His research interest is organic optoelectronic devices. He was awarded the 2007 Ian Macmillan Ward prize from the Polymer Physics Group of Institute of Physics UK, the 2016 Young Achiever award by his Alma Mater the University of Surrey, and was admitted as Fellow of the Royal Society of Chemistry in 2019. $\mathrm{He}$ is an editorial board member of Reports on Progress in Physics.

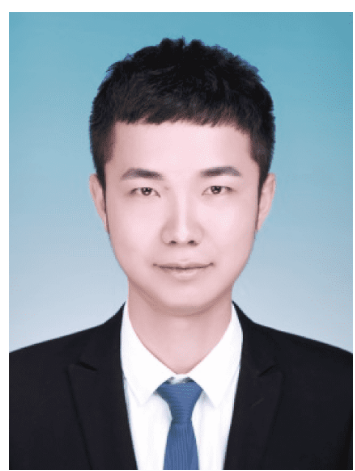

Dr. Xue-Dong Wang is an associate professor at the Institute of Functional Nano \& Soft Materials (FUNSOM) of Soochow University. He received his BS degree in chemistry at Lanzhou University in 2011 and his PhD degree in physical chemistry at the Institute of Chemistry, Chinese Academy of Sciences (ICCAS) in 2016. He also received several awards including the excellent award of president of Chinese Academy of Sciences and the teaching advanced individual of Soochow University. His research focuses on the precise synthesis of organic micro/nanostructures (such as homo-/hetero-structures and superstructures) and organic photonics including organic solid-state lasers and optical waveguides.

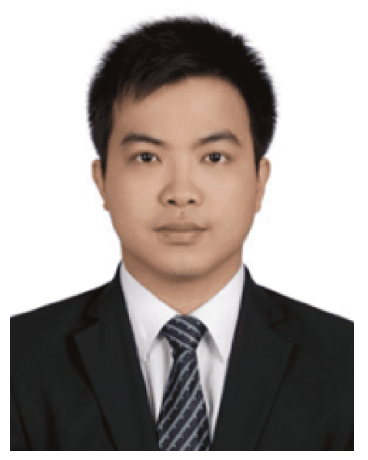

Dr. Sen Xin received his $\mathrm{PhD}$ degree in physical chemistry in 2013 from the Institute of Chemistry, Chinese Academy of Sciences (ICCAS). From 2015 to 2019, he worked as a postdoctoral fellow in the Department of Mechanical Engineering, University of Texas at Austin, under the supervision of Prof. John B. Goodenough. He joined ICCAS as a Professor in 2019. His current research interest focuses on the storage electrochemistry, materials science and electrode-electrolyte interface for advanced energy storage systems including rechargeable $\mathrm{Li} / \mathrm{Na}$-chalcogen batteries and solid-state $\mathrm{Li} / \mathrm{Na}$ batteries. He is now a member of "Young Scientist Committee" of SCIENCE CHINA Chemistry. 


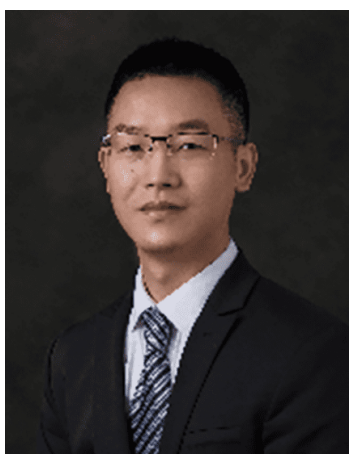

Dr. Da-Gang Yu received his BS degree from Sichuan University in 2007 and PhD with Prof. Dr Zhang-Jie Shi from Peking University in 2012. Then he carried out postdoctoral research with Humboldt fellowship in the group of Prof. Dr Frank Glorius, Muenster University. Since 2015, he has been working independently in Sichuan University with the support from "The Thousand Young Talents Plan", the National Natural Science Foundation of China-Outstanding Young Scholars and Fok Ying Tung Education Foundation. His research interest mainly focuses on the transformation of $\mathrm{CO}_{2}$, radical chemistry and novel transition-metal catalysis. He received Thieme Chemistry Journal Award in 2017, ACP Lectureship Awards to Japan in 2017, and Chinese Chemical Society Youth Award in 2018.

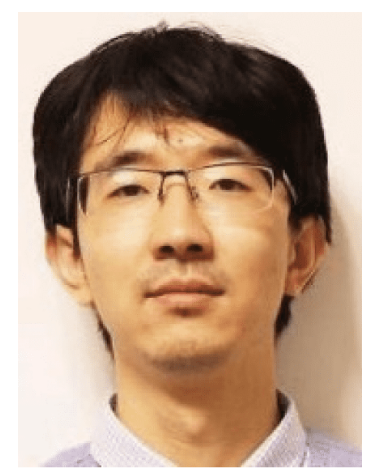

Dr. Yifu Yu received his BS degree and $\mathrm{PhD}$ degree in chemical engineering from Tianjin University. He carried out postdoctoral research in Nanyang Technological University under the direction of Prof. Hua Zhang (from July 2014 to July 2017). Currently, he is a professor in the Institute of Molecular Plus at Tianjin University. His research interest focuses on the artificial nitrogen cycle.

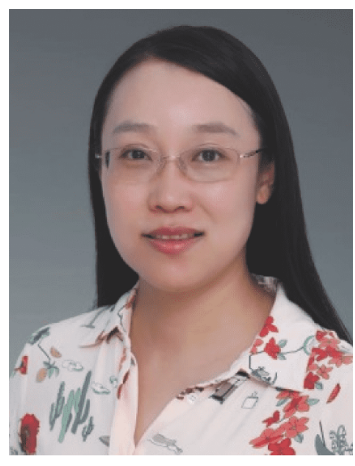

Dr. Zhi Zhu received her BS degree in Chemistry from Peking University in 2006 and her $\mathrm{PhD}$ in Analytical Chemistry from the University of Florida in 2011. After graduation, she joined Xiamen University as Assistant Professor and was promoted to Associate Professor in 2012, and Professor in 2015. Her current research particularly focused on the development of functional nucleic acid probes, microfluidic methods and signal amplification strategies to improve the sensitivity and selectivity of analysis in complex biological system. She is the recipient of the Excellent Youth Scholars of China in 2014, Chinese Chemical Society Young Chemist Award in 2016, Fujian Top Young Innovative Talents in 2016, and "Changjiang Scholar Award Program" young scholars in 2018.

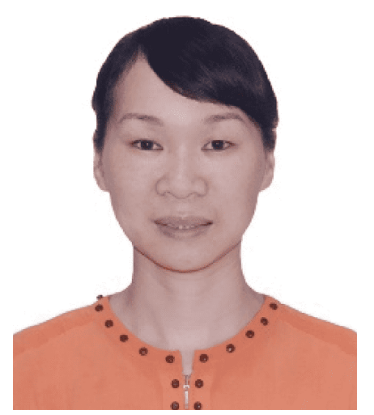

Dr. Yingping Zou received her PhD degree from the Institute of Chemistry, Chinese Academy of Sciences (ICCAS) in 2008, and in the following years she performed her postdoctoral research in Laval University and visiting research in Stanford University. She was promoted to full professor in 2014 in Central South University (CSU). Currently, her researches focus on the organic small molecules/ polymers for high-performance optoelectronic devices. Her group has developed a design strategy defined as “A-DA'DA" to obtain a series of high-performing non-fullerene acceptors (NFAs), which have set new records for the certified power conversion efficiencies in organic solar cells by National Renewable Energy Laboratory (NREL). She has been awarded several prizes, such as J. Mater. Chem. A emerging investigator (2020), Cell press 2019 Chinese annual paper prize, Jingqing chemical excellence award of Chinese Chemical Society (2019), Sunshine outstanding achievement award (2019) and distinguished young scholars of Hunan province (2017). 